\title{
Wind Tunnel Flow Induced Vibration Characteristic Analysis
}

\author{
Zongzheng Liü, , Yan Shi', Zhenhua Chen', Xiqiang Yan', Yisheng Yang ${ }^{1}$ \\ ${ }^{1}$ China Aerodynamics Research and Development Center, Mianyang \\ alzzcd@163.com
}

Key words: Wind tunnel; Flow induced vibration; Characteristic.

\begin{abstract}
Absract: Analysis of wind tunnel flow induced vibration characteristic is to understand the high-speed airflow caused mechanism of flow induced vibration, take appropriate to restrain vibration reducing body piercing vibration circuit. This paper introduces the characteristics of flow induced vibration source, sets up a wind tunnel loop fluid mechanics continuous equation, momentum equation and energy equation, determines the wind tunnel flow movement differential equation, analyzes the mathematical model, puts forward the basic method, establishes the vibration preliminary theoretical basis for experimental study. The results show that source analysis, establishing the mathematical equations and adopting reasonable vibration reduction measures will help improve the theoretical basis of wind tunnel flow induced vibration.
\end{abstract}

\section{Introduction}

The vibration aroused by flow has been highly concerned because of its destructiveness. The purpose of flow-induced-vibration (FIV) research work is to explore the vibration mechanisms, the forces between the research objects and the movement of the research objects inspired by the fluid.

The internal flow and external flow can also arouse the FIV. When the internal flow happens, the fluid is contained in the structure. The typical internal flow is the flow in the fluid-conveying pipeline. When the velocity or pressure of fluid changes, it causes the vibration of the pipeline. The vibration of the pipeline will react on the fluid. This action and reaction is fluid-structure interaction. When the external flow happens, the structure is in contained in the fluid, such as the flow on the surface of the sphere, cylinder, plane wing, and turbine...etc.

Since 1950's, large-scale research work on FIV has been carried out. The FVI is a multi-disciplinary crossing science which is about physics, fluid mechanics, solid mechanics, elastic mechanics and material mechanics etc, so it is difficult to understand all the FIV mechanisms. The reach work is on different level. For example, it have obtained many research achievements on fluid-conveying FIV, while fewer on high speed air induced vibration.

The wind tunnel used in aerodynamics reach realm is a kind of piping-like facility in which the artificial-drived-air runs. During its working, the circuit of the wind tunnel will vibrate fiercely under the force of high speed air. This phenomenon is typical FIV. The vibration will lower the accuracy of test results, influence the performance of the wind tunnel, reduce the life span of the wind tunnel, change the mechanical properties of the key components and sensitive compnents. The researches about the vibration characteristics of the circuit and key components have a great influence on wind tunnel's safty, reliability and efficiency.

The flow in the wind tunnel circuit belongs to the internal flow, while the flow on some inner key components such as compressor blades, valves, model sting, second throat and damping net 
belongs to the external flow. So, the vibration of the wind tunnel is caused by the external and internal flow.

At present, the vibration research on wind tunnel is mainly based on the analysis of structural vibration without considering the interaction with the air flow. In this thesis, the research on the concerning of FVI is to know the vibration mechanism and find the suitable ways for vibration suppression. The research proposed a fundamental method for vibration suppression from the fluid mechanics aspect, include putting forward the basic continuity equation, momentum equation, energy equation successively and finally building up the mathematics model for the internal flow. This research is helpful to understand the fundamental theory of FVI in wind tunnel.

\section{The FVI characteristic in wind tunnel}

The changing of the speed and pressure of the internal flow in a wind tunnel and the vibrating of the structural in it can induce the air pulsation or the circuit vibration. The interaction between the flow and the structure is called fluid-structure coupling, which enlarge the vibration. Four kinds of coupling are include in fluid-structure coupling issue in wind tunnel, which are friction coupling, poisson coupling, junction coupling and wave-wave coupling $[1,2]$.

(1)Friction coupling is induced by viscous friction between circuit wall and flow. In general, dynamic response is weak in the condition of low-frequency friction. When the frequency is higher, plug flow will appear in the boundary layer which make the friction frequency and dynamic characteristic more complicated.

(2)Poission coupling is induced by the interaction between the local air pressure and the wall stress. This coupling have closely relationship with the poison's ratio of the wall material. Poission coupling can influence the flow's dynamic characteristic remarkably. In certain conditions, poission shock can damage the circuit severely.

(3)Junction coupling is induced by the sharply change of the flow in the junction part of the wind tunnel. When the flow passes the corner, transition, throat... etc, the flow become pressure unbalance and the flow impacts the structure severely.

(4)Burden coupling is induced by the elbow pipe whose section is not circle. When the flow passes the pipe, the flow change its direction and pressure. Burden coupling has the trend to make the elbow pipe to become straight.

(5)Wave-wave coupling is induced by the shutting up of the valve in transient wind tunnel. In the shutting process, a series of flow-direction pressure waves move upward to make the upward flow compressed and pressure rising. According aerodynamics-shock-wave theory, when the wave arrives at the reflective boundary first time, the wave is reflected to the opposite direction and pressure drop. The reflected wave is named rarefaction wave. After several loops, the flow evolves into shock-shock interference flow in the wind tunnel.

\section{FVI equation in the wind tunnel}

The equations of fluid motion

The flow in the circuit of the wind tunnel follows three basic fluid mechanics equations: Continuity equation, momentum equation and energy equation ${ }^{[3]}$.

The continuity equation in wind tunnel is 
$\frac{\partial}{\partial t} \oiint_{v} \rho d v+\oiint_{s} \rho v d s=0$

Where $\rho$ is the fluid density, whose unit is $\mathrm{kg} / \mathrm{m}^{3}$;

A is the area, whose unit is $\mathrm{m}^{2}$;

The momentum equation in wind tunnel is

$\int_{s}(\rho v \cdot d s)+\frac{\partial}{\partial t} \oiint_{v} \rho \rho v \cdot d v=\oiiint_{v} \rho f d v-\int_{s} p d s+F_{v i s c o u s}$

Where $\oiiint_{s}(\rho v \cdot d s) v+\frac{\partial}{\partial t} \oiiint_{v} \rho v \cdot d v$ is momentum rate ;

$\oiiint_{v} \rho f d v$ is distributed viscous body force whose unit is $\mathrm{N}$;

$-\oiint_{s} p d s$ is the pressure force, whose unit is $\mathrm{N}$;

$\boldsymbol{F}_{\text {viscous }}$ is the viscous force, whose unit is N.

The energy equation in wind tunnel is

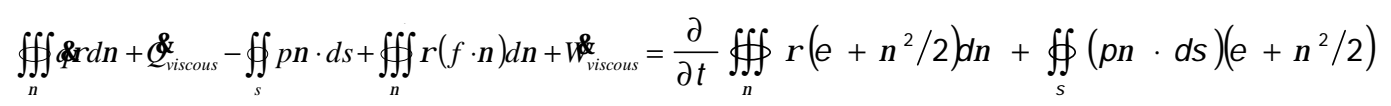

Where $\oiiint_{v}$ of $d v+\Phi_{\text {viscous }}^{\&} \quad$ is heat rate ;

Wiscous is power rate ;

$\frac{\partial}{\partial t} \oiiint_{v} \rho\left(e+v^{2} / 2\right) d v$ is total energy rate ;

$\oiint_{s}(p v \cdot d s)\left(e+v^{2} / 2\right)$ is the lost energy in the control surface.

The momentum differential equation of the flow inside the wind tunnel

The wind tunnel is a typical fluid-transporting pipeline. The momentum differential equation ${ }^{[4,5]}$ of the flow inside it is as follows without considering the fluid compression.

EI $\frac{\partial^{4} w}{\partial x^{4}}+M U^{2} \frac{\partial^{2} w}{\partial x^{2}}+2 M U \frac{\partial^{2} w}{\partial x \partial t}+(M+m) \frac{\partial^{2} w}{\partial t^{2}}=0$

Where $E I$ is the flexural rigidity of the wind tunnel ;

$M$ is the flow mass in unit length;

$m$ is the circuit mass in unit lenth;

$U$ is the average velocity of the flow ;

$\mathrm{w}$ is the displacement at $x$ coordinate in time $t$;

In the equation(11), the four items represent elastic restoring force, centrifugal force, Coriolis 
force and inertia force. The influence to the circuit is as follows:

(1) Centrifugal force has negative stiffness effect on the circuit. The natural frequency of the system will increase as the flow velocity decrease.

(2) Inertia force: the additive mass introduce by inertia force will lower the natural frequency of the circuit of the wind tunnel. When the flow velocity increases to a certain degree, the circuit will become divergence instability.

(3) Coriolis force has great influence on the modal of the circuit of the wind tunnel, as it will make the typical real modal impossible and there is traveling wave component exist in the mode shape; in the certain condition, Coriolis force become negative damping and make the circuit self-excited vibrations happen.

The dimensionless form of the equation(4) is as the follow ${ }^{[4,5]}$

$$
\frac{\partial^{4} \eta}{\partial \xi^{4}}+v^{2} \frac{\partial^{2} \eta}{\partial \xi^{2}}+2 \sqrt{\beta} \frac{\partial^{2} \eta}{\partial \xi \partial \tau}+\frac{\partial^{2} \eta}{\partial \tau^{2}}=0
$$

In the equation(5),

$\eta=\frac{w}{L}, \xi=\frac{x}{L}, \tau=\sqrt{\frac{E l}{M+m}} \frac{t}{L^{2}}, \beta=\frac{M}{M+m}, v=\sqrt{\frac{M}{E I}} L U$

Where $L$ is the length of the circuit of the wind tunnel ;

$\beta$ is the mass ratio;

$v$ is the dimensionless average velocity.

The circuit of the wind tunnel is shell structure, but it is similar to the beam structure and has the similar momentum differential equation. The equation(4) is based on ideal fluid model without considering the viscous force between the flow and the solid wall of the wind tunnel and Non-uniformity velocity in the cross section of the wind tunnel.

Paidoussis's research expresses ${ }^{[5-7]}$ that the friction between the wall and flow and the pressure loss along the circuit will be not appear in the equation because of cancellation. Viscous force will not appear in the equation if considering the circuit and the flow as a whole system.

Because the flow velocity is not uniform along the cross section, centrifugal force and Coriolis force in the equation(4) are need to be calculated based on the velocity and be integral at whole section. Coriolis force is the linear term, and after integral it is same as average $\mathrm{U}$; while Centrifugal force is the quadratic form of the flow velocity, and after integral it is bigger than average U. So, $2 M U$ in equation(4) should be replaced by $\int_{A} \rho u^{2} d A$.

$$
\int_{A} \rho u^{2} d A=\alpha M U^{2}
$$

Then

$\alpha=\frac{\int_{A} \rho^{2} d A}{M U^{2}}=\frac{\int_{A} U^{2} d A}{A U^{2}}$

$\alpha$ is the low Regime correction coefficient of the centrifugal force (ideal fluid, $\alpha=1$ ). The momentum differential equation ${ }^{[4,5]}$ of the wind tunnel circuit is

EI $\frac{\partial^{4} w}{\partial x^{4}}+\alpha M U^{2} \frac{\partial^{2} w}{\partial x^{2}}+2 M U \frac{\partial^{2} w}{\partial x \partial t}+(M+m) \frac{\partial^{2} w}{\partial t^{2}}=0$

The dimensionless form of the equation( 8$)$ is as the follow ${ }^{[4,5}$ 


$$
\frac{\partial^{4} \eta}{\partial \xi^{4}}+\alpha v^{2} \frac{\partial^{2} \eta}{\partial \xi^{2}}+2 v \sqrt{\beta} \frac{\partial^{2} \eta}{\partial \xi \partial \tau}+\frac{\partial^{2} \eta}{\partial \tau^{2}}=0
$$

When $\mathrm{Re}>2300$, the flow in the circuit is turbulent flow, the velocity of the fluid particles along the axis can be divide into average velocity $u$ and fluctuating velocity $u^{\prime}$.

Hence

$$
\begin{aligned}
& \int_{A} u d A=\int_{A} \bar{u} d A+\int_{A} u^{\prime} d A \\
& \int_{A} u^{2} d A=\int_{A} \bar{u}^{2} d A+2 \int_{A} \bar{u} u^{\prime} d A+\int_{A} u^{\prime 2} d A
\end{aligned}
$$

Define equivalent velocity as

$\uplus=\alpha U$

And equivalent mass as

$$
M t=\frac{M}{\alpha},(M++M)=(M+m)
$$

Then accord (8) and(9), we obtain ${ }^{[4,5]}$

$$
\begin{aligned}
& \text { EI } \frac{\partial^{4} w}{\partial x^{4}}+M J^{2} \frac{\partial^{2} w}{\partial x^{2}}+2 M+\frac{\partial^{2} w}{\partial x \partial t}+(M+m) \frac{\partial^{2} w}{\partial t^{2}}=0 \\
& \frac{\partial^{4} \eta}{\partial \xi^{4}}+k^{2} \frac{\partial^{2} \eta}{\partial \xi^{2}}+2 k \sqrt{\beta} \frac{\partial^{2} \eta}{\partial \xi \partial \tau}+\frac{\partial^{2} \eta}{\partial \tau^{2}}=0
\end{aligned}
$$

In the equation (14) and (15)

$$
\begin{aligned}
& k=\sqrt{\frac{M}{E I}} L U=\sqrt{\alpha} \sqrt{\frac{M}{E I}} L U=\sqrt{\alpha \nu} \\
& \beta=\frac{M}{M+m}=\frac{M}{\alpha(M+m)}=\frac{\beta}{\alpha}
\end{aligned}
$$

$\tilde{v}$ and $\tilde{\beta}$ are dimensionless form of the equivalent velocity and equivalent mass.

Through Comparing, we find equation (4) and (14), equation (5) and (15) are the same form. We

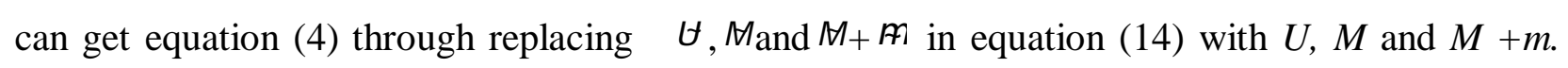
We can also get equation (5) through replacing $k$ and $\beta$ in equation (15) with $v$ and $\beta$.

Considering the axial force, gravity, fluid static pressure, elastic foundation, internal damping of material, external viscous damping and other factors, the linear momentum differential equation of the circuit ${ }^{[5-7]}$ is

$E^{*} I \frac{\partial^{5} W}{\partial x^{4} \partial t}+E I \frac{\partial^{4} W}{\partial x^{4}}+\left\{M U^{2}-T+p A(1-2 v \delta)-\quad\left[(M+m) g-M \frac{d U}{d t}\right](L-x)\right\} \frac{\partial^{2} W}{\partial x^{2}}+2 M U \frac{\partial^{2} w}{\partial x \partial t}+(M+m) g \frac{\partial w}{\partial x}+$ $k w+c \frac{\partial w}{\partial t}+(M+m) \frac{\partial^{2} w}{\partial t^{2}}=0$

$E^{*} I$ is the internal damping coefficient of the material (Kelvin-Voigt viscoelastic model),$c$ is external viscous damping coefficient of the material, $\mathrm{k}$ is Winkler elastic foundation coefficient, when the pipe can move along the axis, $\delta$ is 0 , or $\delta$ is $1, v$ is poission ratio, p is static pressure in the wind tunnel hole, $\mathrm{T}$ is the axis force. 


\section{the vibration reduction analysis of the wind tunnel}

From analysis of the fluid-structure coupling of the wind tunnel, we can find that friction coupling, poisson coupling, junction coupling and wave-wave coupling are the main cause of the FVI. Equation(4) shows that the elastic restoring force, centrifugal force, Coriolis force and inertia force roused by the four couplings have relations with flexural rigidity, flow mass in unit length, the circuit mass in unit lenth,the average velocity of the flow, and the displacement at x coordinate. The equation (18) presents the theory of FVI more comprehensively. We know from it that the FVI also has relation with axial force, gravity, fluid static pressure, elastic foundation, internal damping of material, external viscous damping and etc.

No matter what style are the FVI, there are two ways to reduce the vibration : one is to control the flow's pressure fluctuation; the other is to adjust wind tunnel's natural frequency to avoid the vibration strengthen ${ }^{[8]}$.

(1) Control the flow's pressure fluctuation

a. Changing the flow's pulsation frequency. The flow's pressure fluctuation of the wind tunnel has close relationship with wind tunnel's flowing length and size, the length and position of the branch, the flow temperature...etc.. It is easy to change length and size of the circuit and branch in designs, this can avoid fluid-structure interaction.

b. Adopting suitable vibration reduction measures. The position, size of the damping net, and honeycomb has great influence to the vibration reduction, so it is useful to set reasonable buffer device, such as the damping net, and honeycomb...etc.

(2) Adjust the circuit natural frequency of the wind tunnel.

a. It is useful to avoid the sharp size change of the circuit if the local flow pressure is higher than else.

b. Increasing the circuit's damping of the wind tunnel. The standard practice to increase the circuit's damping is to place the metal spring, rubber or cork in under the fixed support to change the structure's damping. These have the effect of vibration reduction or vibration isolation.

c. Layout optimization of the circuit's supports. We can raise the stiffness of the circuit by laying the circuit's supports reasonably. The Vibration Characteristics will also change by that way. After changing the support layout of circuit, the amplitude will decrease, but the exciting force aroused by flow pressure fluctuation will not change.

In this thesis the flow's differential equations of motion and FVI differential equations are acquired. Researchers can find solutions of the above equations to the specific wind tunnel. Researchers can also use numerical simulation method to solve the equations to find ways to reduce the vibration. This thesis are both theoretically significant and practically valuable for wind tunnel design.

\section{Reference}

[1]Zhang Li xiang, Huang Wenhu, A S TIJSSELING. Review of FSI analysis of fluid-conveying pipes $[\mathrm{J}]$. Hydrodynamic research and progress , 2000 ( 9 ) : 366-379.

[2]Li Yanhua. Study on Vibration and Noise and Its Characteristics of Pipeline Systems Considering Fluid-Structure Interaction [D]. Harbin: Engineering university in Harbin,2011.

[3]Xu Huafang. Aerodynamicist foundation [M]. Peking: Defence industry publisher,1979. [4]Guo Changqing. Flow-Induced Vibration and Instability of Pipes Conveying Fluid and Plates in Axial Flow [D]. Peking:Tsinghua university,2010. 
[5]Paidoussis M P. Fluid-Structure Interactions: Slender Structures and Axial Flow, Vol.1.London: Academic Press, 1998.

[6]Paidoussis M P. The canonical problem of the fluid-conveying pipe and radiation of the knowledge gained to other dynamics problems across applied mechanics. Journal of Sound and Vibration, 2008, 310(3):462-492.

[7]Paidoussis M P, Li G X. Pipes conveying fluid: a model dynamical problem. Journal of Fluids and Structures, 1993, 7(2):137-204.

[8]Yu Jiafu, Ma Tingxia, Zhong Kui, Wang Hailan, Zhu Yaming, Zhao Xiao. The Hazard Analysis and Improving Measures in Cleaning-up Operations of Three-phase Separator of Zhongyuan Oilfield [J]. College of science and technology college journal in Chongqing, 2015 ( 2 ) : 85-91. 